\title{
Modification of the Adams Clasps to be Ball Clasps in Clinical Practice Saves Laboratory Time and Makes the Treatment Process of Functional Appliance Smoother
}

\author{
Xiaolei $\mathrm{Hu}^{1,2,4 \#}$ and Xiaomian $\mathrm{Wu}^{1-3 \# *}$ \\ ${ }^{1}$ Chongqing Key Laboratory of Oral Diseases and Biomedical Sciences, College of Stomatology, Chongqing, Medical University, \\ Chongqing, China
}

${ }^{2}$ Chongqing Municipal Key Laboratory of Oral Biomedical Engineering of Higher Education, College of Stomatology, Chongqing Medical University

${ }^{3}$ Department of Orthodontics, College of Stomatology, Chongqing Medical University

${ }^{4}$ Key Laboratory of Clinical Laboratory Science, Ministry of Education, College of Laboratory Medicine, Chongqing Medical University

${ }^{\#}$ These authors contributed equally to this work.

*Corresponding author: Xiaomian Wu, Chongqing Key Laboratory of Oral Diseases and Biomedical Sciences, College of Stomatology; Chongqing Municipal Key Laboratory of Oral Biomedical Engineering of Higher Education, College of Stomatology; College of Stomatology, Department of Orthodontics, College of Stomatology, Chongqing Medical University, Chongqing, China,

Email:wuxiaomian@hospital.cqmu.edu.cn; wuxiaomian898@163.com

\section{ARTICLE INFO}

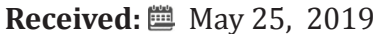

Published: 蔧 May 31, 2019

Citation: Xiaolei Hu, Xiaomian Wu. Modification of the Adams Clasps to be Ball Clasps in Clinical Practice Saves Laboratory Time and Makes the Treatment Process of Functional Appliance Smoother. Biomed J Sci \& Tech Res 18(3)-2019. BJSTR. MS.ID.003165.

\begin{abstract}
Twin-block appliance (TB) is a powerful appliance to treat the dental and maxillary protrusion in children aged 8-14 years who have transitional dentition. And if the producing process of TB is delayed and/or the children's teeth erupt, the TB could not match with the dentition. This paper offered a simple method to modify the Adams clasp to be Ball clasp in clinical. This method could save much clinical and laboratory work and time and offer powerful retention for the TB to make the treatment process smoother by fully using the undercut of the teeth. This is important for increasing the compliance of functional appliance treatment and vital for the success of TB.
\end{abstract}

Keywords: Twin-block appliance; Functional appliance; Adams clasp; Ball clasp; Compliance

\section{Introduction}

The protrusion of maxillary bone and/or maxillary incisor not only affects dentofacial appearance but also increase the risk of incisor trauma. What's more, the abnormal dentofacial appearance has a bad effect on the children's self-confidence development and a negative social experience. So, kinds of functional appliances are designed and improved to treat these malocclusions. Twinblock (TB) is one of them and is widely used in United Kingdom [1]. TB is improved by lots of studies to treatment these kinds of malocclusions more effectively2-5. In China, TB was improved to be Sagittal Guidance Twin-block appliance (SGTB), which was composed of a mandibular removable component and a bonded maxillary counterpart [2]. Ball clasps between the mandibular incisors and Adams clasps on the first premolars and the first molars offer the retention for the mandibular removable part. The angulation of the inclined surface in the SGTB was changed from $45^{\circ}$ to $70^{\circ}$ which could change the force vector to be more sagittal and encourage the patient to close lips naturally. In most time, children receive TB treatment at the age of 8-14 years old with transitional dentitions [3-6]. While the producing of TB is time consuming, and if the producing process is delayed in the busy season of dental laboratory center, which submerged with orders, the children's transitional dentition will change due to tooth eruption. Or children's tooth erupts during the TB treatment. The TB will not match the changed dentition. In this paper we reported a simple method to make this process smoother, by offering the protocol, the mechanism under this method and the case reports. 


\section{Methods and Results}

\section{Case 1}

A 13-year-old boy complained of maxillary incisors protrusion with an overjet more than $8 \mathrm{~mm}$ in the early permanent dentition. The patient agreed to receive two phases treatment and the first phase will have TB treatment. The TB was producing within one week and matched well with the mandibular dentition (Figure 1).

\section{Case 2}

A girl, aged 11 years old, complained of maxillary protrusion with an ANB angle of $6.8^{\circ}$ and a transitional dentition. She accepted two phases treatment plan and had the first phase I of TB. Unfortunately, the producing of TB met the busy season of dental laboratory, and it took one month. When the patient came back to the orthodontics, her mandibular primary tooth lost, and the permanent tooth erupted. While the dentition changed, the mandibular part of TB could not match with the mandibular dentition (Figures $2 \& 3$ ). Regularly, it required the orthodontics to get the dental cast of the patient and have the occlusion record of the patient again. And the dental laboratory center had to produce the new TB for the patient. All of these will cost more than one week, and a lot of laboratory and clinical work was required (Figures $2 \& 3$ ).

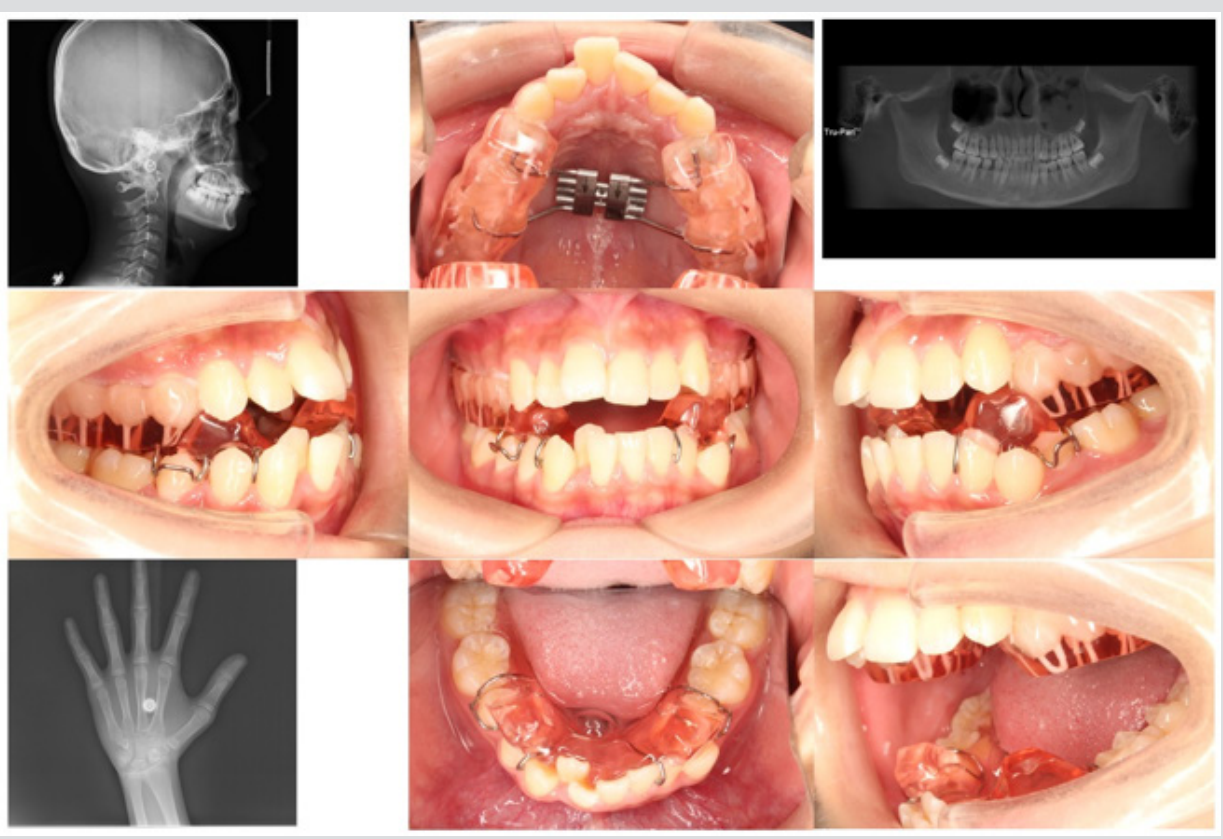

Figure 1: Twin-block appliance (TB) match well with the early permanent dentition of a 13-year-old boy.

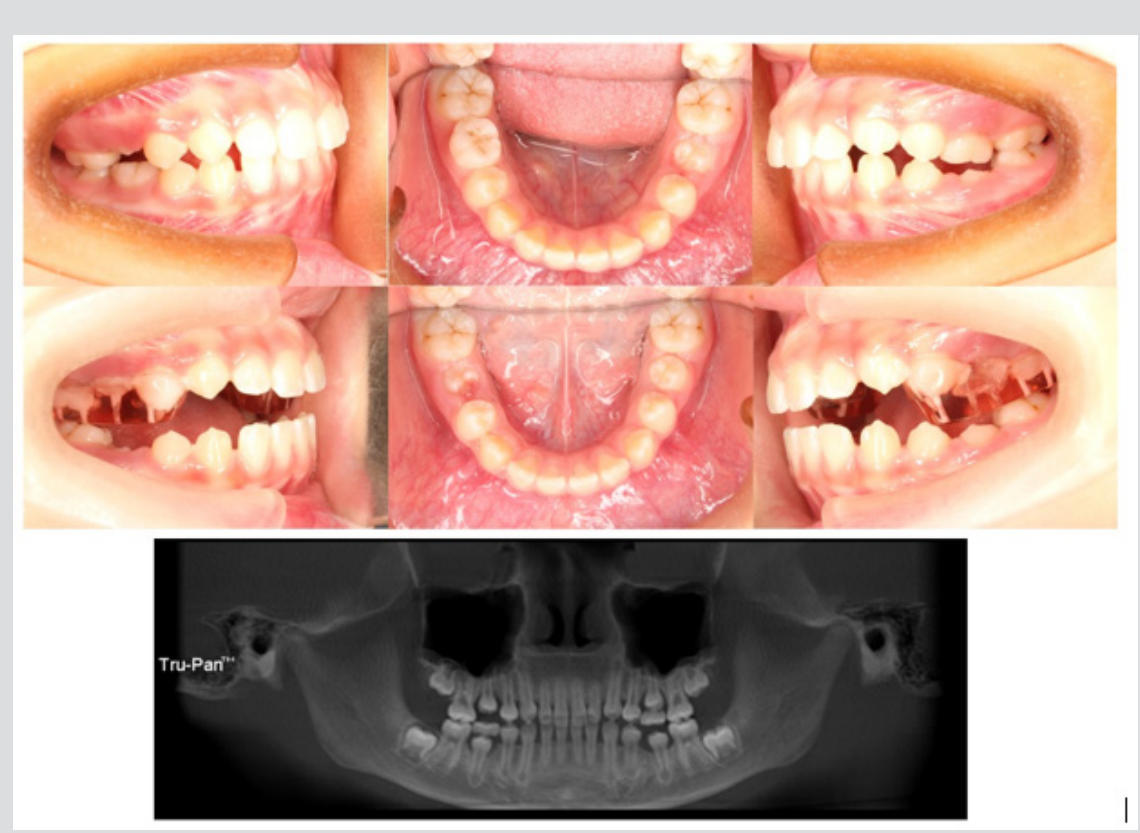

Figure 2: The Comparison of the dentition change in the mandible of the patient. 


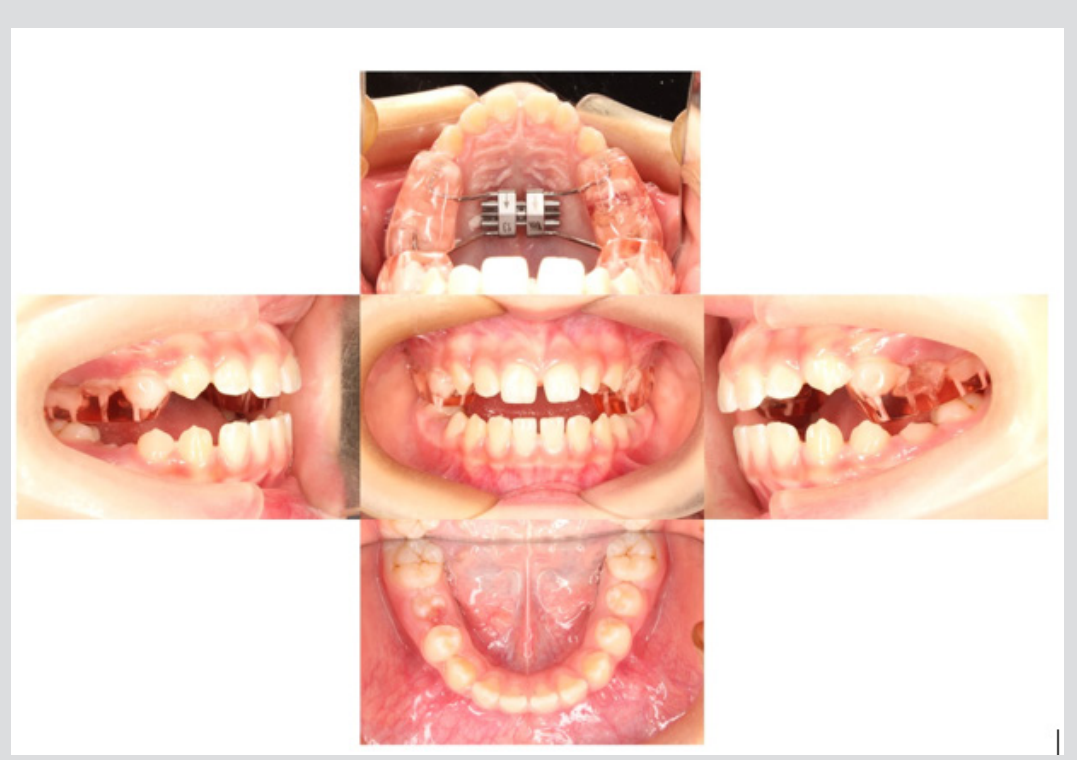

Figure 3: The mandibular part of TB could not match with the dentition in the mandible.

After carefully analyzing the change in the early permanent dentition in the patient, it was easy to find the changed parts located in the premolar. This was due to the eruption times and sequence of permanent teeth. The Adams clasps in the mandibular part of TB still pointed to the undercut of the premolar. How to make full use of the undercut? We directly cut the beam in the Adams clasp and changed the Adams clasp to be Ball clasp, and carefully ground the end to be smooth as the mimic mechanism shown Figure 4. While the Ball clasp was more flexible, the Ball clasp could get to the undercut easier and offered a powerful retention for the TB (Figure 5). This directly modification in clinical practice could make the treatment process smoother and save clinical and laboratory work (Figures 4 \& 5).

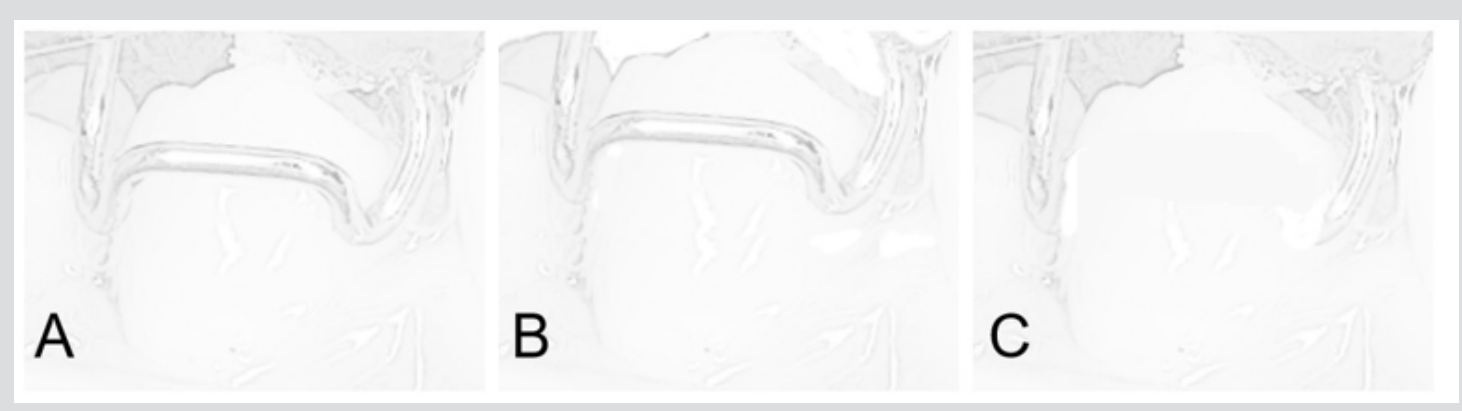

Figure 4: The mimic mechanism of change the Adams clasp to be Ball clasp and relationship of the undercut in the premolar and the Adams clasp and Ball clasp.

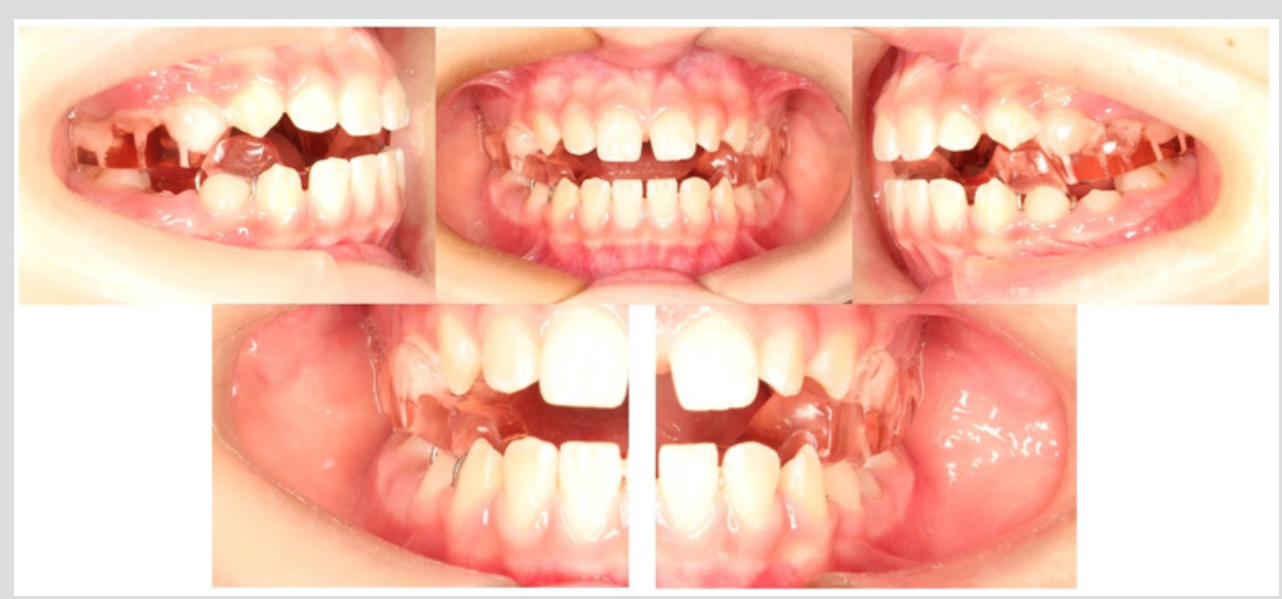

Figure 5: After change the Adams clasp to be Ball clasp, the Ball clasp makes fully use of the undercut and offers powerful retention for the TB and the TB matched well with mandibular dentition. 


\section{Discussion}

Twin-block appliance is a powerful appliance to treat the dental and maxillary protrusion [2,6-8]. It's effective is not limited in the prevention of the dental and maxillary protrusion, but also has a favorable effect in vertical growth and horizontal growth in the temporomandibular joint region [8]. Wear time is important for the effectiveness of TB on dental and skeletal changes [5]. The compliance with removable functional appliances is crucial for the success of the functional treatment. And perceived treatment progress is one of vital factors influencing compliance with removable functional appliance [1]. The delay of TB producing or having dental cast too many times will reduce the compliance and cause the failure of treatment. In this study, we offered a protocol to modify the Adams clasp to be Ball clasp in clinical practice which was convenient and effective to make the treatment process of TB smoother. Both Adams clasps and Ball clasps are powerful retention for functional appliance, but there are some differences between the two clasps. On the one hand, with the help of beam in the Adams clasp, the Adams clasp is more stable, but the beam also reduces the flexibility of the Adams clasps. On the other hand, the Ball clasp is more flexible compared to Adams clasp. So, cut the beam from the Adams clasps will make the clasp more flexible to fully use the undercut of the premolar and offer a powerful retention for the functional appliance.

\section{Acknowledgment}

This study was supported by the National Natural Science Foundation of China 31400808, 81570979 and 21402018. It was also supported by Natural Science Foundation Project of Chongqing, China (CSTS2015JCYJA10039) and Science and Technology Research Project of Chongqing Municipal Education Commission of China (KJ1600226) and Program for Innovation Team Building at Institutions of Higher Education in Chongqing in 2016 (CXTDG201602006).

\section{References}

1. El Huni A, Colonio Salazar FB, Sharma PK, Fleming PS (2019) Understanding factors influencing compliance with removable functional appliances: A qualitative study. American Journal of Orthodontics and Dentofacial Orthopedics 155(2): 173-181.

2. Li P, Feng J, Shen G, Zhao N (2016) Severe Class II Division 1 malocclusion in an adolescent patient, treated with a novel sagittal-guidance Twinblock appliance. American Journal of Orthodontics and Dentofacial Orthopedics 150(1): 153-166.

3. Parkin NA, McKeown HF, Sandler PJ (2001) Comparison of 2 modifications of the twin-block appliance in matched class II samples. American Journal of Orthodontics and Dentofacial Orthopedics 119(6): 572-577.

4. O Brien K, Wright J, Conboy F, Appelbe P, Davies L, et al. (2009) Early treatment for Class II Division 1 malocclusion with the Twin-block appliance: A multi-center, randomized, controlled trial. American Journal of Orthodontics and Dentofacial Orthopedics 135(5): 573-579.

5. Parekh J, Counihan K, Fleming PS, Pandis N, Sharma PK (2019) Effectiveness of part-time vs full-time wear protocols of Twin-block appliance on dental and skeletal changes: A randomized controlled trial. American Journal of Orthodontics and Dentofacial Orthopedics 155(2): 165-172.

6. O Brien K, Wright J, Conboy F, Sanjie Y, Mandall N, et al. (2003) Effectiveness of early orthodontic treatment with the twin-block appliance: A multicenter, randomized, controlled trial. Part 1: Dental and skeletal effects. American Journal of Orthodontics and Dentofacial Orthopedics 124(3): 234-243.

7. Miyazawa K, Sakai N, Tsutsui T, Tabuchi M, Goto S (2015) The simplified bite jumping appliance: An improved functional appliance. Orthodontic Waves 74(1): 18-21.

8. Doshi UH, Mahindra R (2014) Effective temporomandibular joint growth changes after stepwise and maximum advancement with Twin Block appliance. Journal of the World Federation of Orthodontists 3(1): 9-14.

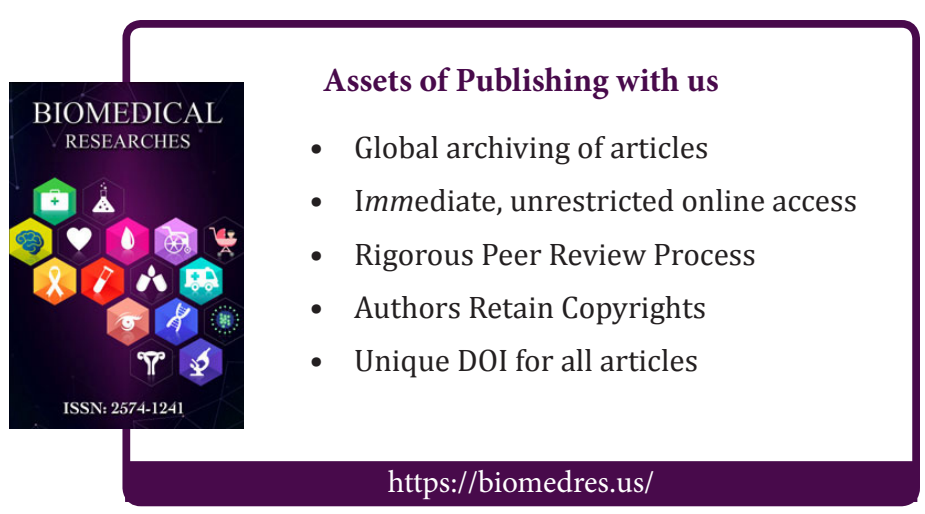

